\title{
Parental Control Technologies und die Überwachung kindlicher Mobilität
}

\begin{abstract}
Sarah Berg
Jan Wehrheim

Im Kontext fortschreitender (auch urbaner) Technisierung und Digitalisierung etabliert sich ein stetig wachsender Markt an Tracking-Anwendungen, die es Eltern ermöglichen, den Standort ihrer Kinder in Echtzeit zu erfahren und ihre Bewegungen nachzuverfolgen. Die bloße Verfügbarkeit dieser als Parental Control Technologies verhandelten Technologien erklärt aber noch nicht ihre tatsächliche Nutzung. Auch ob sie Technologien der Kontrolle und Überwachung von Kindern und Jugendlichen sind, erklärt sich nicht aus ihnen selbst, sondern wird in konkreten Praktiken interaktiv und machtvoll ausgehandelt. Der Artikel formuliert Thesen zu den Hintergründen der Verbreitung raumbezogener Parental Control Technologies sowie zu ihren Folgen und fragt, welche stadt- und raumforscherischen Implikationen diese haben können.
\end{abstract}

Ersteinreichung: 30. November 2020; Veröffentlichung online: 26. November 2021

An English abstract can be found at the end of the document.

Sogenannte Parental Control Technologies (PCT) stellen eine neue Variante der in Gegenwartsgesellschaften boomenden Technologien dar. In der sich in der Bezeichnung ausdrückenden Perspektive geht es um Techniken, zum Beispiel Apps, die es im Kern Eltern oder anderen Nutzer_innen erlauben, Aktivitäten von Kindern und Jugendlichen nachzuvollziehen respektive zu überwachen. Wir wollen im Folgenden einen Einstieg und Überblick über jüngere technische Entwicklungen in Bezug auf PCT liefern und Thesen zu den Hintergründen ihrer Verbreitung sowie zu deren Folgen formulieren. Dabei interessieren uns insbesondere raumbezogene PCT und damit deren stadtund raumbezogene Implikationen für kindliche und jugendliche Mobilität. Zwei miteinander verbundene Dimensionen sind dabei zu unterscheiden, die jeweils mit den in der Anwendung entstehenden Daten zusammenhängen: die (Mit-)Konstruktion von (Stadt-)Gesellschaft und Räumlichkeit durch Massendaten sowie der Einfluss der Technologien auf soziale Beziehungen und Aktivitäten der konkreten Nutzer_innen.ZZunächst erfolgen jedoch einige basale Überlegungen zu den jüngeren Entwicklungen der Digitalisierung von Gesellschaft, denn Parental Control Technologies stellen nur eine kleine Neuerung unter vielen dar und sie sind gleichzeitig in ihrer Relevanz nur in Verbindung mit weiteren Entwicklungen versteh- und erklärbar. 


\section{Die Digitalisierung von Gesellschaft und drei Prämissen zur Einordnung von PCT}

Technische sind nicht von gesellschaftlichen Entwicklungen zu trennen. Sie verlaufen vielmehr in Wechselwirkungen, das heißt, weder resultiert aus gesellschaftlichen „Problemen“ automatisch eine Suche nach technischen Lösungen, noch determinieren neue Technologien, wie und wofür sie genutzt werden, und somit auch nicht gesellschaftliche Entwicklungen (zur Einführung vgl. etwa Degele 2002). Gesellschaften sind zudem keine homogenen sozialen Gebilde, sondern vielmehr als Beziehungen zwischen unterschiedlichen Akteur_innen mit unterschiedlichen Deutungen und Interessen zu denken. Diese Überlegungen sind auch für das Verhältnis von Technisierung und Digitalisierung zu Überwachung zu berücksichtigen, denn Überwachung ist kein analytisch präziser Begriff. Er wird vor allem normativ verwendet: Nicht jegliche Tätigkeit des gegebenenfalls systematischen Beobachtens oder des Erhebens von Daten wird als Überwachung verhandelt. Seine Brisanz erlangt der Begriff erst dann, wenn entsprechende Prozesse beziehungsweise Erwartungen, was aus ihnen folgen könnte, kritisiert werden.[1] Ob Prozesse der Digitalisierung und Technisierung von Gesellschaft und sozialen Beziehungen zu Überwachung führen und dies weitere Veränderungen nach sich zieht, wie es auch die Bezeichnung Parental Control Technologies nahelegt, ergibt sich also nicht unmittelbar aus Digitalisierung und Technisierung selbst.

Dies gilt auch für den Trend zur Digitalisierung von Stadt und für den Boom an verräumlichten Datensätzen und raumbezogenen Technologien. Die zentralen Entwicklungen sind ganz überwiegend aber keine städtischen oder auch nur raumbezogene Phänomene, sondern sie werden lediglich auch in Bezug auf Stadt und Raum relevant. Entsprechende Bezüge lassen sich vielfältig finden: von massenhaften Videokameras, die vermeintliche Kriminalitätsschwerpunkte beobachten, über E-Tickets im ÖPNV und Google Maps, das den Weg zum nächsten Restaurant im Verhältnis zum eigenen Standort oder den freien Parkplatz anzeigt, bis zur sensorbestückten Hauswand, die Graffiti noch während des Sprühens melden soll. Mobilitäts- und Ortsbezüge werden jedoch deshalb besonders relevant, weil Bewegungen und Räume als Chiffre für soziale Phänomene und Beziehungen interpretiert werden und damit gleichzeitig vom Sozialen abstrahiert wird (vgl. grundsätzlich Belina 2013).

Mindestens drei Aspekte sind dabei für die wissenschaftliche und politische Diskussion von Stadt und Gesellschaft in Bezug auf Digitalisierung und die dabei entstehenden Daten grundlegend relevant. Erstens sind die entstehenden Daten potenziell alle miteinander verknüpfbar, und dazu gehören auch alle Daten, die sonst noch produziert werden: durch das Surf- und Kaufverhalten, die Nutzung von Zoom oder Lernplattformen wie Moodle, Handystandortdaten, Gesundheitsdaten, digitale Steuererklärungen, Bilddateien bei Einwohnermeldeämtern, Flughäfen oder Instagram und so weiter und so fort. Zweitens determinieren die intendierten Nutzungen nicht die tatsächlichen: Apps für Smartphones können praktisch sein oder Spaß machen; insbesondere jedoch kostenlose, aber kommerzielle Apps sammeln vor allem Daten und greifen dabei nicht selten auch auf Bilder 
oder Kontaktlisten zu, die auf den Handys gespeichert sind.[2] Moodle und andere Systeme ermöglichen es, (universitäre) Lehre mehr oder weniger brauchbar online abzuhalten, und zeigen Lehrenden, wann welche Studierenden sich letztmalig eingeloggt haben. Die Techniken bieten somit immer mehr Funktionen als eventuell geplant war oder als sichtbar sind. Was dabei die manifeste und was die latente Funktion ist, zeigt sich oft erst mit der Zeit und variiert mit den Perspektiven und damit ebenso, welche Funktionen intendiert programmiert wurden - also ob etwa das Sammeln von Daten Ziel oder Nebeneffekt ist. Drittens sind alle Daten geeignet, sowohl Einzelpersonen über sehr wenige Kombinationen zu identifizieren, selbst wenn diese vermeintlich anonym sind (vgl. Rocher et al. 2019; de Montjoye et al. 2015), als auch in Massendaten Muster zu erkennen, Personenkategorien statistisch zu generieren und soziale Prozesse zu beeinflussen - seien sie als Steuerung intendiert oder das Nebenprodukt von Algorithmen.

Gary T. Marx (2006) hat deshalb die Unterscheidung zwischen hard und soft surveillance eingeführt. Ersteres umschreibt Prozesse, die früher mit dem Tragen von Schlapphüten assoziiert wurden oder heutzutage etwa als IMSI-Catcher[3] daherkommen; Zweiteres meint die Potenzialität der Überwachung, die nicht als solche empfunden wird. Es muss eben kein_e Polizist_in ununterbrochen neben einem herfahren, um zu erfassen, wo sich jemand bewegt, solange das Smartphone (mit oder ohne WLAN-, Bluetooth- oder GPS-Aktivierung) eingeschaltet ist. Kevin D. Haggerty und Richard V. Ericson (2006) wiederum sprechen von function creep, um zu verdeutlichen, wie zu bestimmten Zwecken eingeführte Technologien und deren Daten (einzeln oder in Kombination mit anderen) geradezu proliferierend für andere Zwecke genutzt werden. Damit verbunden sprechen sie von einer surveillance assemblage: Es ist - international durchaus variierend - nicht mehr (nur) der orwellsche Big Brother, sondern eine Vielzahl höchst unterschiedlicher Akteur_innen und Systeme, die mit ihren Datenproduktionen und (potenziellen) Nutzungen ineinandergreifen, und als Überwachung deutbare Aktivitäten werden auch in privaten Beziehungen relevanter. Überwachung, so wiederum Shoshana Zuboff (2018), ist zu einem Motor und Kernelement des spätmodernen Kapitalismus geworden.

Trotz der drei genannten Aspekte und ihrer Einordnungen müssen die einzelnen technischen Neuerungen zunächst für sich betrachtet werden. Aus ubiquitärem Computing und ubiquitärer Digitalisierung lässt sich nicht ableiten, warum eine konkrete Neuerung wie PCT genutzt wird und was daraus folgt. Das „Zeitalter des Überwachungskapitalismus“ (Zuboff 2018) muss im interaktiven Handeln erst hervorgebracht werden. Technologien werden in der Praxis mit Bedeutungen gefüllt und erst so (in der Summe und in ihren Verbindungen) gesellschaftlich relevant.

\section{Beobachtete Kindheit und Jugend: Parental Control Technologies}

Unter der Abkürzung PCT werden zahlreiche Anwendungen und Geräte gefasst, die auf unterschiedliche Bereiche des elterlichen oder kindlichen und jugendlichen Lebens zielen. Wie Marx und Valerie Steeves (2010) ausführen, beginnt diese Bandbreite im weiteren Sinne bereits vorgeburtlich mit der 
Möglichkeit des Zyklus- und Ovulationstrackings oder pränataler Diagnostik und setzt sich später dann als akustische oder häufig auch videobasierte Überwachung des - zumeist schlafenden - Babys oder Kleinkinds über das sogenannte Babyphone fort. Für Kinder und Jugendliche existieren viele weitere Produkte, vom vernetzten und abhörenden Spielzeug[4] über private Drogentests bis hin zu Tests auf sexuelle Aktivität (vgl. ebd.). Am meisten etabliert und ausdifferenziert sind jedoch zum einen sogenannte Jugendschutz-Apps, die auf Aktivitäten von Kindern und Jugendlichen im Internet zielen, die Nachverfolgung und das Blockieren spezifischer Webseiten oder des Internetzugangs zu spezifischen Zeiten sowie das Lesen der online verschickten Nachrichten ermöglichen; zum anderen sind es die hier interessierenden raumbezogenen Parental Control Technologies. Diese nutzen Ortungstechnologien wie Global Positioning System (GPS) oder Radio-Frequency Identification (RFID) zur zeitgleichen oder zeitversetzten Nachverfolgung der kindlichen/jugendlichen Aufenthaltsorte durch die Eltern. Dazu sind die Kinder mit einem entsprechenden Gerät ausgestattet, das zum Beispiel in einen Anhänger, eine Armbanduhr/Smartwatch oder den Schulranzen integriert, aber auch in Kleidungsstücke eingenäht oder als Anwendung auf dem Smartphone des Kindes installiert sein kann. Die Eltern können nun auf ihrem Computer oder Smartphone je nach Funktionsumfang der entsprechenden Technologie live den Standort ihres Kindes auf einer Karte beobachten und bei Bewegung verfolgen (Tracking) oder sich rückwirkend die Historie der Aufenthaltsorte anzeigen lassen (Tracing; vgl. Gryl 2019). Viele der Technologien sind über diese Basisfunktion der räumlichen Ortung hinaus noch mit Zusatzfunktionen ausgestattet. Häufige Bestandteile sind zum Beispiel Notrufknöpfe und -funktionen oder das „Geofencing“, das es den Eltern erlaubt, spezifische Orte und Bereiche als „sichere“ und „gefährliche“ beziehungsweise erlaubte und verbotene Orte zu definieren und einen Alarm bei Verlassen und Betreten der jeweiligen Bereiche einzurichten. So können beispielsweise das eigene Zuhause oder die Schule als sicher definiert werden, sodass Eltern eine Benachrichtigung über ihr Smartphone erhalten, wenn ihr Kind zu Hause oder in der Schule angekommen ist. Umgekehrt können Orte wie viel befahrene Straßen, vermeintliche Kriminalitätsschwerpunkte, Industriebrachen, Rotlichtviertel oder Aufenthaltsorte „falscher Freunde“ als verboten markiert werden, sodass die Eltern in Echtzeit eine Warnung erhalten, wenn ihr Kind diese dennoch betritt. Welche Orte entsprechend ausgeschlossen werden, variiert mit den lokalen Gegebenheiten und deren Bewertungen durch die Nutzer_innen der PCT.

Der Markt für diese raumbezogenen Parental Control Technologies ist bereits groß, weit ausdifferenziert und stark wachsend: Die international verfügbare Tracking-App „Findmykids“ wurde den Angaben im Google Play Store zufolge bis Oktober 2020 bereits über zehn Millionen Mal heruntergeladen, die Anwendung „Life360“ sogar über 50 Millionen Mal, zudem mit circa einer Million Bewertungen. Das Play-Store-interne Ranking gibt als die drei erfolgreichsten Apps in der Kategorie „Eltern“ drei Parental Control Technologies an, die alle mindestens GPS-Ortung und Geofencing erlauben, zum Teil darüber hinaus auch Kontrollen der Handynutzung wie eine sekundengenaue Zeitleiste der kindlichen App-Nutzung oder eine automatische Erkennung „verdächtiger“ Fotos („FamiSafe“). Während „Life360“ 
vor allem in den USA große öffentliche Bekanntheit erlangt hat,[5] ist der Markt für ähnliche Anwendungen auch in Deutschland fest etabliert: Die Nutzungszahlen der deutschen Anwendung „Familonet“ stiegen innerhalb von vier Jahren von rund 500.00o (vgl. Kistler 2015) auf circa 2,5 Millionen im Jahr 2019 (vgl. Maas 2019).

Für Wearables und eigenständige Devices, die im Gegensatz zu oben genannten Anwendungen auch dann genutzt werden können, wenn das Kind (noch) kein eigenes Smartphone besitzt, gelten ähnliche Einschätzungen: Eine Suche beim Online-Versandhandel Amazon liefert für die Suchbegriffe „Kinder“ und „GPS“ über 3.00o Treffer - von Anhängern bis zur KinderSmartwatch -, die vielfach auch mit deutschsprachigen Bewertungen versehen sind. Der Mobilfunkanbieter Vodafone brachte im Jahr 2018 eine eigene Smartwatch heraus, die mit den Smartphones der Eltern verknüpft werden kann und explizit für Grundschulkinder beworben wird (vgl. Baumann 2018).[6] International werden solche Geräte als GPS-Tracker oder RFID-Tags mit unterschiedlichen Verbreitungsgraden etwa zur Kontrolle von Anwesenheitszeiten bereits an Schulen in den USA, Brasilien (Ema/ Fujigaki 2011; Donath 2012), Japan, Südkorea (Ema/Fujigaki 2011) und Schweden (Nihlén Fahlquist 2017) eingesetzt. In Deutschland machte die in einen Schulranzen integrierte „Schutzranzen-App“ über angekündigte Kooperationen unter anderem mit den Städten Wolfsburg (dort wurde die Kooperation nach massiver Kritik durch Datenschützer_innen mittlerweile wieder abgesagt; vgl. Greiner/Horchert 2018) und Ludwigsburg Schlagzeilen (vgl. Ludwigsburger Kreiszeitung 2018).

\section{Vorstellungen kindgerechter Räume}

Hinsichtlich der Funktionen solcher PCT und deren Anwendung ist das Grundprinzip elterlicher Kontrolle der kindlichen Wege und Aufenthaltsorte kein neues und die Ausgestaltung der Differenzierung zwischen kindgerechten und nicht kindgerechten Räumen ist dabei keineswegs eindeutig. Bereits die Vorstellung, es gäbe überhaupt so etwas wie Räume für Kinder - und damit auch solche, die für Kinder nicht geeignet sind -, ist keine historische Kontinuität, sondern vielmehr ein Produkt der Moderne. Erste Voraussetzung dafür war eine Perspektive auf Kindheit als eigenständige Lebensphase, die sich im 17. und 18. Jahrhundert herausformte - mit einem damit einhergehenden Bildungsverständnis, in dem das Lehren und Lernen an Bedeutung gewann und Kinder zunehmend Gegenstand - auch staatlicher erzieherischer Zugriffe wurden (vgl. Schreiber 2018; Wucherpfennig 2019).

Im Zuge der Industrialisierung und der damit einhergehenden Trennung (männlicher) Lohnarbeit und (weiblicher) Haushaltsarbeit etablierte sich schließlich auch die ideologische Unterscheidung öffentlicher und privater Sphären, die dadurch ebenfalls jeweils geschlechtlich kodiert sind (vgl. Strüver 2018). Mit ihren Müttern und deren Sorgearbeit werden auch die Kinder zunehmend in die private Sphäre verwiesen - und in raumbezogenen PCT wird dies nicht zuletzt über die Markierung des eigenen Zuhauses als „sicherer Ort“ im Rahmen der Geofencing-Funktion (re-)produziert.

Thematisierungen kindgerechter Räume und damit einhergehende Problematisierungen von unbeaufsichtigter kindlicher Mobilität waren 
jedoch von Beginn an zusätzlich milieu- beziehungsweise klassenspezifisch konnotiert. Als Folge von Armut und Ungleichheit wurden die sich neu etablierenden Kinderzimmer zunächst nur Teile des bürgerlichen Wohnens und auch die Aufsicht der Kinder durch Eltern oder Hausangestellte war entsprechenden Haushalten vorbehalten. Auf den Straßen der großen, industrialisierten Städte waren vor allem Kinder aus dem Proletariat präsent, was wiederum als Indikator für Erziehungsrisiken und für eine defizitäre, klassenspezifische Sozialisation als Gefährdung sozialer Ordnung gedeutet wurde. Es ging also um Gefahren für Kinder und Jugendliche - durch Gewalt, Verführung, Verkehr - und um Gefahren durch Kinder und Jugendliche, die durch die Straßensozialisation „Kriminalität“ erlernten oder gesellschaftliche Moralvorstellungen unterminierten.

Die mittlerweile zu Klassikern avancierten Diagnosen einer „Verhäuslichung“(Zinnecker 1990) oder einer „Verinselung“(Zeiher 1990)von Kindheit legen eine Verstärkung und Ausdifferenzierung von „Zonen der Sichtbarkeit und Unsichtbarkeit von Kindern" (Schreiber 2018: 309) in der Stadt nahe. Sie sind jedoch nicht unumstritten: Zwar wird auf die genannten Thesen der Verhäuslichung und Institutionalisierung von Kindheit, die zunehmend an gezielt für Kinder eingerichteten Orten und in Bildungseinrichtungen stattfinde, vielfach Bezug genommen, gleichzeitig wird jedoch aus einer sozialisations- und bildungstheoretischen Perspektive auch die Bedeutung der Zwischenwege für Kinder betont und auf deren eigensinnige Perspektiven und Praktiken verwiesen (vgl. Egger/Hummel 2016). Doch nicht nur die Frage, $o b$ es spezifisch (nicht) kindgerechte Räume gibt, wurde historisch unterschiedlich beantwortet. Auch die Vorstellungen davon, wie diese Räume für Kinder auszusehen haben, unterliegen gesellschaftlichen und historischen Wandlungsprozessen. So changiert in der Pädagogik die Bewertung der Straße zwischen gesellschaftlichem Lernfeld und „Ort des Verderbens“ (vgl. Fegter/Andresen 2019; Behnken/Zinnecker 2019).

Diese kontroversen Perspektiven auf die Straße als (kein) Ort für Kinder verweisen auf die Funktionen raumbezogener Parental Control Technologies - Schutz (vor den Gefährdungen von Straße und Großstadt), Fürsorge und Kontrolle - sowie auf die fließenden Übergänge von „care and control“ (Lyon 2001). Dabei ist das Räumliche als Chiffre für Annahmen zu sozialen Phänomenen, Beziehungen und mit ihnen assoziierten Risiken zu lesen. Die „Straße“ ist diesbezüglich das Symbol für alle Räume außerhalb der Sichtbarkeit und Kontrolle von (als vertrauenswürdig gedeuteten) Erwachsenen - elterliche Wohnung, Heim, Schule, Sportverein, Kindergarten, Jugendzentrum.[7] PCT sind neue technische Optionen, mit den genannten Ambivalenzen und variierenden Problematisierungen umzugehen.

Das Technische der PCT ist jedoch nicht das einzige Neue und Strategien der Beaufsichtigung von Kindern verändern sich. Einige Eltern entscheiden sich für das sogenannte Chauffeuring und bringen ihr Kind mit dem Fahrrad, dem Auto oder zu Fuß zur Schule, zu Freund_innen oder zum Musikunterricht, um so den sicheren Weg zu garantieren (vgl. Fotel/Thomsen 2004; Carver et al. 2013). Michael Feldhaus (2003) untersuchte Anfang der 200oer Jahre, wie und warum Eltern Handys nutzen, um die Aufenthaltsorte und Abwesenheitszeiten ihrer Kinder zu erfragen, zu kontrollieren und (neu) auszuhandeln. Entsprechende Praktiken führten, so Carol 
Barron (2014, 2017), auch zu teilweise subversiven Umgangsstrategien der Kinder und Jugendlichen mit dieser elterlichen Kontrolle über Telefonate und Kurznachrichten. Der Unterschied zwischen der Handynutzung der frühen 20ooer Jahre und dem Einsatz von Smartphones mit entsprechenden PCTApps liegt zuvorderst in der Intentionalität: Bringen Handys nur das Potenzial der Kontrolle (und permanenten) Erreichbarkeit mit sich und produzieren gegebenenfalls erst im Gebrauch den Verdacht der kindlich-jugendlichen Abweichung, zielen PCT explizit auf (auch unbemerkte/unverhandelte) Beobachtung. Der Unterschied zum „Chauffeuring“ wiederum liegt in der Distanz und somit der „Unsichtbarkeit“ der Beobachtung sowie in den Daten, die über die Nutzung entstehen.

Die Frage ist nun, wie es dazu kommt, dass Eltern verstärkt zu Parental Control Technologies greifen. Die schlichte Existenz der technischen Möglichkeit reicht als Erklärung nicht aus.

\section{Thesen zu Gründen der Verbreitung von PCT}

Der PCT-Boom fällt zunächst in den erwähnten allgemeinen Boom der Technisierung und Digitalisierung. Immer mehr Bereiche des Lebens werden technisch organisiert und gemanagt und jenseits von Open-Source-Produkten werden die entsprechenden Techniken ökonomisch interessiert beworben - wobei Gewinne aus Verkaufs-/Abonnementpreisen und/oder über die Sammlung und den Verkauf der entstehenden Daten generiert werden. Unabhängig von Gebrauchswerten der Techniken für ihre Nutzer_innen erscheint diese Entwicklung ,alternativlos“.[8] Insofern ist es naheliegend, dass Digitalisierung und Technisierung auch in Eltern-Kind-Beziehungen Einzug halten, wenn entsprechende Produkte verfügbar sind. Dies fällt jedoch in eine Phase gesellschaftlicher Transformation, die zur Interpretation der Nutzung und Verbreitung von PCT mitzudenken ist. Aktuelle Gesellschaften vor allem des globalen Nordens werden als neoliberale und neosoziale beschrieben, die zudem durch gesellschaftliche Ausdifferenzierung gekennzeichnet sind.

$\mathrm{Zu}$ nennen sind unter anderem deregulierte Arbeitsmärkte und - damit zusammenhängend - Änderungen bei wohlfahrtsstaatlichen Arrangements, die auf die jeweiligen Haushalte respektive Familien wirken. Dabei ist insbesondere die mit Ende des sogenannten Normalarbeitsverhältnisses teilweise erzwungene, teilweise im Kontext von weiblicher Emanzipation erwünschte Verbreitung von Doppelverdiener_innenhaushalten relevant. Zudem nimmt die Anzahl von Haushalten Alleinerziehender zu. Beide Familienkonstellationen bringen veränderte Herausforderungen in Bezug auf die Betreuung, Versorgung und Aufsicht von Kindern mit sich, die nur begrenzt durch Kitas, Ganztagsschulen oder sozial differenziert durch Au-pair-Mädchen kompensiert werden (vgl. Jurczyk et al. 2009). Der generalisierte Anspruch an Eltern, sich um ihre Kinder „adäquat“ zu kümmern, und der stigmatisierende Vorwurf, Kinder gar „verwahrlosen“ zu lassen, wenn diese Teile des Tages ohne Betreuung sind, betrifft also nicht mehr ausschließlich unterprivilegierte Eltern, sondern es sind immer breitere Teile der Mittelschichten damit konfrontiert, das „Problem“ der Kinderbetreuung und der Sorge um die Kinder zu lösen. Politische und sozioökonomische Rahmenbedingungen bleiben in dieser individuellen 
Responsibilisierung, für die PCT potenziell Teillösungen anbieten, jedoch außen vor: „None of the technologies - no matter how strange or impractical - offer anything more than micro-scale and private solutions to what are social and political-economic problems. Of course, in the contemporary neoliberal climate, that is precisely their allure." (Katz 2001: 49)

Solche objektivierbaren Hintergründe treffen neben der Verfügbarkeit der PCT ihrerseits auf veränderte Diskurse und Leitbilder im Zusammenhang mit der grundsätzlichen Ambivalenz von Fürsorge zwischen Versorgung, Sicherheit, Bildung und Autonomie. Zum einen geht es um Entwicklungen, die als Securitization oder Versicherheitlichung gefasst werden, also darum, immer mehr Bereiche des alltäglichen Lebens aus der Perspektive sicher/ unsicher zu betrachten, und daran anknüpfende oder dem vorausgehende politische Programmatiken (vgl. Krasmann et al. 2014). Datensammlungen und Überwachungstechnologien werden typischerweise mittels Unsicherheitsdiskursen durchgesetzt. PCT können dann als Artefakte des spätmodernen Sicherheitsdispositivs begriffen werden. Sie versprechen, Aufenthalte und Bewegungen und damit soziale Aktivitäten transparenter zu machen, also neues Wissen zu produzieren und damit über Kontroll- und Interventionsmöglichkeiten (das spätere Gespräch mit der Tochter, der Ruf der Polizei etc.) Unsicherheiten oder auch nur Unsicherheitsgefühle zu reduzieren. Insbesondere Geofencing-Funktionen ermöglichen es, „unverantwortliches“ Verhalten von Kindern und Jugendlichen durch „verantwortliche“ Eltern sichtbar zu machen.

Bei den raum- und bewegungsbezogenen PCT geht es zudem um die Verortung von Risiken, wobei Apps auch neues „Wissen“bereitstellen und so die Anlässe ihrer Nutzung mitproduzieren und gleichzeitig die Risiken über die Möglichkeit räumlichen Vermeidungsverhaltens beherrschbar erscheinen lassen. So zeigt der Marktführer „Life360“ in den USA auch „local threats in your area“, also „Crime Spots“ oder Wohnorte registrierter „sex offender“ auf den Karten an. Darüber hinaus fügen sich PCT in Sicherheitsstrategien, die auf Prävention setzen. Trends zur Technisierung und zur potenziell unbegrenzten Risikovorsorge greifen ineinander. In Bezug auf Kinder und Jugendliche bewegen sich diese Risiken auf zwei Ebenen: erstens der Sorge um mögliche Gefahren sowie darum, die eigene Fürsorge und Verantwortung angemessen gewährleisten zu können; und zweitens auf der Ebene des Risikos von Abweichung - und damit des Strebens nach Regulierung insbesondere jugendlicher Identität und jugendlichen Verhaltens.

Merkmale von Un-Sicherheitsdiskursen sind dementsprechend nicht nur staatliche Programme, vielmehr wird die Verantwortung für die eigene Sicherheit im neoliberalen Staat zunehmend an die Bürger_innen delegiert und marktförmig organisiert (vgl. Goold et al. 2010; Katz 2001). Mit dieser Kommodifizierung werden Sicherheits- und Kinderschutzprodukte zu Gütern - und in ihrer Vermarktung und ihrem Erwerb spielen Schutz, Angst und Sicherheit eine große Rolle. So zeigen Benjamin Goold et al. (2010: 9 f.), wie bereits der Akt des Erwerbs von Sicherheitsprodukten das Gefühl bringen kann, etwas selbst in die Hand genommen zu haben, und so Beruhigung ermöglicht. Beworben wird Sicherheit dementsprechend weniger im Kontext von Kontrolle als vielmehr von Selbstschutz: Hilfe per Knopfdruck rufen zu können (etwa familo.net/de). Gleichzeitig sind die Gründe für den Konsum 
jedoch vielfältig und gehen deutlich darüber hinaus: Sicherheitsprodukte können Gegenstände von Moden, also „cool“ sein, und sie haben eine spezifische Ästhetik, die in die Konsumentscheidung einbezogen wird. Als Konsumgüter fungieren sie auch als „key marker of social identity and belonging“ (ebd.: 7) und sind so Mittel des Ausdrucks von Subjektivität, sozialer Hierarchie und Distinktion.

Der in Deutschland dominierende Anbieter familo.net wirbt entsprechend mit einer Multifunktionalität, die gleichzeitig vielfältige Be-Deutungen der App erlaubt: „Kommunikation, Freiheit, Sicherheit“(familo.net/de) und auch die umgekehrte Richtung der Standort- und Bewegungsbestimmung bietet: Standorte von Eltern und Geschwistern können mit angezeigt und Gruppen zusammengestellt werden. Auch wird bei der Produktpräsentation mit der Einstellung von „Lieblingsorten“ begonnen, nicht mit „gefährlichen Orten“. Je nach Funktionalitätsumfang und Bewerbung werden also unterschiedliche Möglichkeiten und Wirkungen nahegelegt, die in der Anwendung erst hergestellt werden müssen.

Aber auch wenn man den Analysen zur Versicherheitlichung und den Thesen einer „politics of fear“ (Altheide 2009) oder eines „paranoid parenting“ (Furedi 2002) folgt, zeigen sich in der Literatur konkurrierende Annahmen: So argumentiert Jessica Nihlén Fahlquist (2017: 124), die Nutzung von PCT erlaube es Eltern, ihre Angst zu managen. Demgegenüber konstatieren Marx und Steeves (2010), dass die Bewerbung der Technologien einen geradezu appellativen Charakter entfalte, weil sie nicht nur vielfältige Risikolagen für Kinder und Jugendliche präsentiere, sondern auch Eltern (und Schulen) spezifisch adressiere: „parents and schools simply cannot ,afford to allow children to interact without surveillance because of the proliferating, risks that must be managed and controlled" (ebd.: 214).

Die Versicherheitlichung korrespondiert insofern zum anderen mit der neoliberalen Betonung von Eigenverantwortung und damit mit dem, was als Leitbild responsibilisierter Elternschaft diskutiert wird. Responsibilisierung bedeutet hier, Eltern nicht mehr nur für den Schutz ihrer Kinder etwa vor körperlicher Versehrung in die Verantwortung zu nehmen, sondern auch für ihre optimale Entwicklung und Förderung (vgl. Richter/Andresen 2012; Jergus etal. 2018). Die Anrufung an Eltern, eigenverantwortlich und präventiv tätig zu werden, ordnet sich dabei in allgemeine wohlfahrtsstaatliche Entwicklungen aktivierender Sozialpolitik ein (vgl. Lessenich 2008). Inhaltlich sind Verhaltenserwartungen dabei an Idealen und Lebensweisen der Mittelschicht orientiert und die somit milieuspezifische Kritik am Erziehungsverhalten erweist sich vielmehr als Kritik habitueller Unterschiede beziehungsweise als „Sanktionierung von Lebensstilen, ungleichen Ressourcen und gesellschaftlichen Machtverhältnissen als defizitäre Verhaltensmuster" (Kutscher 2008: 40). Zu fragen ist damit auch, ob die Nicht-Nutzung von PCT prospektiv als defizitäre und mangelhafte Verantwortungsübernahme gedeutet oder empfunden wird.

Parental Control Technologies können somitals Instrumente der Ausübung elterlicher Eigenverantwortung im Zuge veränderter wohlfahrtsstaatlicher Arrangements und damit einhergehender normativer Vorstellungen von Elternschaft gelesen werden. Eltern und insbesondere Mütter sind gerade bei gleichzeitiger Berufstätigkeit verstärkt herausgefordert, den Anforderungen 
eigenverantwortlich zu genügen. In diese Familiensituationen und Normenkomplexe treten nun Parental Control Technologies und - so Feldhaus (2003) für Handynutzung zur elterlichen Kontrolle - mit dem Wissen um diese (neuen) technologischen Möglichkeiten tritt zugleich die Verpflichtung zu ihrer Nutzung im Rahmen der verantwortungsvollen Erziehung. PCT ordnen sich damit in generelle Entwicklungen der Mediatisierung von Familie und das remote parenting, also die Gestaltung von Elternschaft über räumliche Distanz, ein (vgl. Kamin/Meister 2020; Ponte et al. 2018).

\section{Thesen zur Nutzung von PCT und deren Folgen}

Verantwortete Elternschaft und etwa arbeitsmarktgetriebenes remote parenting verweisen nicht nur auf neue Anforderungen, Elternschaft zu gestalten. Es stellt sich auch die Frage, welche Implikationen dies auf räumliche Aktivitäten und Strukturen hat. Überlegungen zur Nutzung von PCT und deren Folgen können damit in zwei Dimensionen diskutiert und in Bezug auf Stadt und Raum analysiert werden: einerseits hinsichtlich des „Wissens“, das die Apps und Wearables für die Nutzer_innen produzieren, und andererseits hinsichtlich der Daten, die PCT für die Hersteller_innen und für Dritte produzieren, die wiederum auf sozialräumliche Wirklichkeiten rückwirken und diese verändern können. Der Forschungsstand zu PCT ist bislang überschaubar, aber es werden diverse und teilweise konträre Befunde und Thesen diskutiert. Gleichzeitige ambivalente Effekte sind naheliegend.

So hat bereits die Forschung zu Mobiltelefonen gezeigt, dass diese nicht nur der elterlichen Fernkontrolle dienen, sondern auch als Mittel, mit dem die familiäre Beziehungsgestaltung (vgl. Röser 2007: 136 f.) im Allgemeinen und die Organisation funktionaler Abläufe im Besonderen erleichtert wird (vgl. Feldhaus 2003). Insofern liegt die Annahme nahe, dass PCT zwischen Eltern und Kindern nicht nur ein potenzielles Kontrollverhältnis etablieren, sondern auch neue Möglichkeiten zur Beziehungsgestaltung eröffnen, innerhalb derer wiederum Sicherheitsinteressen zwischen „care and control“ (vgl. auch Fotel/Thomsen 2004; Taylor/Rooney 2017) verschwimmen. So gaben in einer Studie des Deutschen Instituts für Vertrauen und Sicherheit im Internet (2015: 59) 21 Prozent der befragten Eltern an, mittels Mobiltelefon die Sicherheit und Erreichbarkeit auf dem Schulweg zu gewährleisten und fernmündliche Absprachen zu treffen.

Mit und durch Technologien, die über räumliche Aktivitäten informieren, deutet sich zudem eine andere Konnotation an, die den Fokus gerade nicht auf Überwachung legt: Durch das potenzielle Monitoring der Bewegung könnten neue Freiräume wie etwa längere Spiel- oder Ausgehzeiten, Mobilität ohne elterliche Begleitung und erweiterte Aktivitätsradien verhandelt werden (Feldhaus 2003; Williams/Williams 2005). Gerade die Kontaktier- und Ortbarkeit könnte paradoxerweise zu Autonomiegewinnen bei Kindern und Jugendlichen führen, wie es Susann Fegter (2014: 28) auf Basis ihrer Untersuchung zum Frankfurter Bahnhofsviertel für Mobiltelefone und genderspezifisch für Mädchen beschreibt: Wege könnten dadurch ohne Begleitung von Erwachsenen erfolgen (vgl. Ponte et al. 2018; Lachance 2020). Die Deutung von PCT als „Erziehung aus der Distanz“ sowie - aus der Perspektive von Kindern und vor allem von Jugendlichen - teilweise als „lazy 
parenting“ (vgl. Ghosh et al. 2018) kann wiederum als Ausdruck bemängelter Vernachlässigung oder Verlust des Raums für subjektiven Austausch[9] interpretiert werden, ist aber auch dahingehend sinnhaft, dass Jugendliche wegen so gedeuteter elterlicher Bequemlichkeit widerständige Praktiken entwickeln und solche Freiräume von Face-to-face-Begleitung nutzen: Das über die PCT produzierte Wissen, das an die Eltern übermittelt wird, beschränkt sich eben primär darauf, wo sich eine Person (bzw. ihr Smartphone, ihre Jacke oder ihr Ranzen) zu einer bestimmten Zeit befindet oder wohin sie sich bewegt. Was sie dort tut, bleibt unbekannt beziehungsweise wird aufgrund von mit den Orten verbundenen Erwartungen sozialer Phänomene assoziiert. Die potenzielle Kontrollintensität ist eine andere im Vergleich zum elterlichen Chauffeurdienst. Das „helicopter parenting“ (LeMoyne/ Buchanan 2011) ist mit PCT nur ein technisiertes und so zunächst von geringerer Intensität. Freiheits- oder Autonomiegewinne für Kinder und Jugendliche sind örtlich jedoch auf die Räume beschränkt, die ihnen in den machtvollen Eltern-Kind-Aushandlungen zugestanden werden.

Die Möglichkeiten dessen, was Eltern über ihre Kinder auch unabhängig von einer freiwilligen Informationspreisgabe wissen können, verändern sich und das Wissen bleibt Gegenstand von interaktiven Aushandlungspraktiken. Innerhalb sozialer Beziehungen kann aber gerade die Bereitschaft des (partiellen) Nicht-Wissens und damit ein Entgegenbringen von Vertrauen als wesentliche Grundlage für die Gewährung von Privatsphäre gesehen werden (Drüeke et al. 2007; Kühne 2019: 147 ff.). Eine weitere Frage hinsichtlich der Nutzung von PCT wäre also, ob sie zusätzlich oder anstelle anderer Kontrollpraktiken oder Wissensquellen eingesetzt werden und wie dies von den Beteiligten und gegebenenfalls involvierten Dritten Geschwister, andere Eltern, Lehrer_innen etc. - gedeutet wird: als care oder control, als Schutz oder als Überwachung oder nur als Bequemlichkeit - und damit, ob die „Vertrauensfrage“ überhaupt relevant wird. Vor diesem Hintergrund erweist sich technologisch mediierte Kontrolle also auch als Frage des Eingriffs in die Freiheitsrechte von Kindern und Jugendlichen sowie in ihre Möglichkeiten, Selbstbestimmung und -verantwortung zu erlernen und über räumliche Mobilität bisher unbekannte Erfahrungen zu machen, die jedoch nicht aus den Technologien selbst resultieren, sondern erst Ergebnis verhandelter Praktiken im Kontext konkurrierender Anforderungen an Elternschaft wären: „The ideals of protecting a child and promoting their independence can come into conflict with each other. Using technology to track children puts their potential conflict to the fore: How do we protect our children without impeding their growth as individuals?" (Nihlén Fahlquist 2017: 125)

Die Anwendungen von PCT folgen unterschiedlichen Rationalitäten, die erst in diskursiver Praxis wirkmächtig werden. Pragmatistisch und interaktionistisch argumentiert (vgl. grundsätzlich: Blumer 1981), entstehen die Be-Deutungen erst im Gebrauch. Es entsteht jedoch nicht nur für Eltern anderes Wissen über ihre Kinder (oder in den selteneren Fällen vice versa). Die den PCT immanenten Datenproduktionen bieten Potenziale jenseits der Eltern-Kind-Beziehungen. Über die Verbreitung dieser Apps und Wearables und der massenhaft dabei erhobenen beziehungsweise „nebenbei“ entstehenden Daten sind diese in ihrer gesellschaftlichen Relevanz zu 
denken. Greift man den US-amerikanischen Marktführer „Life360“ heraus, so verweist er in seinen Geschäftsbedingungen und der Privacy Policy auf diverse Kooperationspartner_innen und vielfältige Datenerhebungen, die für die eigentlichen Funktionen irrelevant sind.[10] Diese werden mit verschiedenen Partnerfirmen für Werbezwecke - zu denen explizit auch Facebook und Twitter gehören -, aber etwa auch mit Kfz-Versicherungen geteilt (die App beinhaltet auch eine Funktion, die sich auf die elterliche Kontrolle des Fahrverhaltens von Jugendlichen bezieht) und gegebenenfalls an Strafverfolgungs- und andere staatliche Behörden weitergegeben. (Bewegungs- und Aufenthalts-)Daten von Kindern und Jugendlichen können dann in die anfangs angedeuteten „Datenpools“ einfließen und Auswirkungen etwa auf ein „Scoring“ haben, das erst im späteren Leben relevant wird: individualisierend, wenn es bei Versicherungen oder Polizeibehörden um Zugehörigkeiten zu Risikokategorien oder um tatsächliche Aktivitäten der PCT-Träger_innen geht, und damit gleichzeitig statistisch und über die prospektive Steuerung gesellschaftlicher Prozesse. Gerade bei Daten zu Aufenthaltsorten geht es um die statistische Aggregation auf unterschiedlichen räumlichen Maßstabsebenen, aus denen heraus dann Rückschlüsse auf aktuelle oder zukünftige soziale Prozesse oder Ereignisse gezogen werden sollen.[11]

Damit ist anzunehmen, dass PCT sowohl für ihre direkten Nutzer_innen als auch gesellschaftlich Wechselwirkungen in Bezug auf Vorstellungen sozialräumlich verfasster Strukturiertheit von Städten haben werden. So liegen bei lernenden Apps etwa selbstverstärkende Effekte nahe, wenn Gegenden, die oft mit Geofencing belegt werden, als neue „gefährliche Gegenden“ in die Karten der Apps eingespeist würden und so wiederum neues Geofencing nahelegten. Das Image von als gefährlich, amoralisch oder wie auch immer stigmatisierten Orten dürfte sich jenseits sonstiger Informationen verstärken und über Wirkungen auf Mobilität „reale“ Erfahrungen in dort verorteten Situationen unterminieren. PCT wären dann Teil einer technischen Konstruktion „gefährlicher Räume“. Auch könnten sich Ausprägungen (kleinräumlicher) Segregation verstärken und Möglichkeiten, den Umgang mit Differenz zu lernen, reduzieren, wenn eine „Verinselung“ und damit auch Bewegungen zwischen den Orten zunehmend technisch vorinformiert und gezielt stattfinden.

\section{Fazit}

Ob Technologien wie PCT Technologien der Kontrolle und Überwachung von Kindern und Jugendlichen sind oder werden, resultiert nicht aus den Technologien selbst. Entscheidend sind die konkreten Praktiken der Überwachung, die im Handeln interaktiv entstehen und machtvoll verhandelt werden, sowie die Bewertung dieser Praktiken. Parental Control Technologies verweisen daher nicht auf prinzipiell neue Konstellationen in Bezug auf das Verhältnis von Gesellschaft und Kindheit/Jugend einerseits und ElternKind-Beziehungen und Elternschaft andererseits. Die Raum- und Mobilitätsbezogenheit der Apps und Wearables führt die mit der Industrialisierung und Urbanisierung entstandene Unterscheidung zwischen Räumen für Kinder und Räumen, die nicht für Kinder gedacht werden, technisiert fort. Sie modifizieren so die Praktiken der Kontrolle und damit die Formen ihrer 
Aushandlungen und ihrer Wirkungen. Wie entsprechende Veränderungen in der Praxis hergestellt werden und was daraus folgt, ist noch offen. Parental Control Technologies können allerdings bereits jetzt als Technologien gelesen werden, die über die Digitalisierung neue Potenziale entfalten, die über Eltern-Kind-Beziehungen hinausgehen und sich in konkrete gesellschaftliche Entwicklungen fügen: veränderte Anforderungen an und veränderte Thematisierungen von Elternschaft einerseits und einen datengetriebenen Überwachungskapitalismus andererseits.

Dieser Artikel wurde durch den Open-Access-Publikationsfonds der Universität Duisburg-Essen gefördert.

\section{Endnoten}

[1] Die Richtung der Kritik hat sich jedoch verändert. Zielte der Begriff etymologisch zunächst darauf, zu bezeichnen, dass jemand zu lange gewacht hat und damit die Tätigkeit ineffektiv wurde, wird nun die Tätigkeit an sich kritisiert.

[2] Dabei ist zu berücksichtigen, dass es z. B. bei Internetnutzungen um eine Vielzahl von Daten für eine Vielzahl späterer Nutzer_innen geht (bei der Lektüre eines SpiegelOnline-Artikels etwa erfolgen oft viele Hundert Zugriffe von Externen): IP-Adresse und darüber grober Standort, Browser, Bildschirmgröße, Computerhersteller, Betriebssystem, Verweildauer, zuvor besuchte Seiten, Häufigkeit des Besuchs etc. - und dies nicht nur vom Seitenbetreiber, sondern von sämtlichen Anbieter_innen verlinkter oder eingebetteter Seiten.

[3] IMSI-Catcher simulieren Mobilfunkzellen und erlauben es so, Informationen auf SIM-Karten auszulesen, Telefongespräche mitzuhören oder zu unterbinden und den Standort des Mobilfunkgeräts exakter zu bestimmen, ohne dass die Besitzer_innen dies einfach bemerken können.

[4] Die „Hello Barbie“ beispielsweise ist mit Mikrofon, Spracherkennung und Konversationssoftware ausgestattet und kann auf Fragen und Sätze des mit ihr spielenden Kindes reagieren. Über eine Cloud können die Eltern auf die aufgezeichneten und gespeicherten Gespräche ihres Kindes mit der Puppe zugreifen (vgl. Mattel 2015; Praschl 2015).

[5] Im Sommer 2019 wurde „Life360“ zu einem verbreiteten Meme auf der Social-MediaPlattform TikTok und kurz darauf zu einem intensiv diskutierten Gegenstand auf der Plattform Reddit (vgl. Ohlheiser 2019). Die Online-Debatten zogen große Aufmerksamkeit auf sich, unter anderem die des CEO der App, Chris Hulls, der im Folgenden medienwirksam unter Beteiligung jugendlicher TikTok-Nutzer_innen eine neue Funktion zur (vermeintlichen) Erhöhung der Privatsphäre einführte (vgl. Perez 2020).

[6] Im Jahr 2020 wurde das firmeneigene Repertoire durch den „smarten“ Tracker „Curve“ erweitert, der neben GPS auch Bluetooth, Mobilfunk und WLAN zur Standortbestimmung verwendet und bewusst neutral vermarktet wird: „[...] etwa für die Verfolgung von Hunden, Wertsachen wie Schul- oder Laptop-Taschen und Autos“ (Hoepken 2020a). Zudem läuft gemeinsam mit dem Disney-Konzern die Entwicklung einer neuen, noch „smarteren“ „Kids-Watch“ (Hoepken 2020b).

[7] Michael Schetsche hat die sich verändernden Konjunkturen der Problematisierung, ihrer Akteur_innen und Deutungsmuster und damit auch der Verortung sexualisierter Gewalt gegen Kinder herausgearbeitet: Wurde diese zunächst als „Triebverbrechen“ durch Fremde und außerhalb von Familie, Zuhause oder anderen Institutionen verortet, folgten - auch im Zuge von Emanzipationsprozessen - deren Verortung zunehmend in den Familien und Deutungen als „Missbrauch“ durch Väter (Schetsche 1993: 64). In jüngerer Zeit richten sich Problematisierungen verstärkt auf außerfamiliale Institutionen wie Sportvereine, Heime oder Kirchen.

[8] So wird teilweise selbstverständlich beklagt, die Digitalisierung der Schulen sei „verschlafen“ worden, ohne überhaupt darzulegen, welchen Mehrwert die Digitalisierung für Lernprozesse außerhalb von „Pandemiezeiten“ hat. 
[9] „According to the discourses of the teens, the questions asked by those parents in these kinds of situations were not ,how are you?', ,where are you?' or even ,who are you hanging out with?' but rather ,are you still reachable?' and ,will you answer me when I contact you?'.“ (Lachance 2020: 357)

[10] Für Kalifornien etwa benennt das Unternehmen explizit diese Datenarten: „Identifiers, such as your name, address, phone number, email address, unique device identifiers or other similar identifiers; California customer records, such as birth date and payment information; Commercial information, such as records of services purchased, obtained or considered; Internet/Network information, such as device information, logs and analytics data; Geolocation data, such as precise location data from your mobile device (e.g., GPS coordinates) or generated based on your IP address; Inferences about your interests and preferences, generated from your use of our services; and Other personal information, such as driving event and movement data collected from your mobile device, or content or messages you send through the Service.“ (https://support.life36o.com/hc/en-us/ articles/360043228154-Full-Privacy-Policy; letzter Zugriff am 11.8.2021)

[11] Beim sogenannten predictive policing bzw. über den Bezug zu räumlichen Einheiten beim Geopolicing etwa geht es darum, über die Verbindung von Massendaten Ereignisse zu prognostizieren und räumlich spezifisch zu polizieren (Frers et al. 2013).

\section{Autor_innen}

Sarah Berg hat Soziale Arbeit studiert und arbeitet zu Sozialer Arbeit in der Stadt, abweichendem Verhalten und Kontrolle.

sarah.berg@uni-due.de

Jan Wehrheim ist Sozialwissenschaftler und Entwicklungspolitologe. Seine Arbeitsschwerpunkte sind Stadt- und Devianzsoziologie sowie neue Kontrolltechnologien.

jan.wehrheim@uni-due.de

\section{Literatur}

Altheide, David (2009): The Columbine shootings and the discourse of fear. In: American Behavioral Scientist 52/10, 1354-1370.

Barron, Carol (2014): „I had no credit to ring you back“. Children's strategies of negotiation and resistance to parental surveillance via mobile phones. In: Surveillance \& Society 12/3, 401-413.

Barron, Carol (2017): „Where are you, who are you with, what are you doing?“. Children's strategies of negotiation and resistance to parental monitoring and surveillance via mobile phones. In: Emmeline Taylor / Tonya Rooney (Hg.), Surveillance Futures. Social and ethical implications of new technologies for children and young people. London/New York: Routledge, 110-121.

Baumann, Janette (2018): V-Kids Watch: Neue Vodafone Smartwatch verbindet Dich mit Deinen Kindern. https://www.vodafone.de/featured/inside-vodafone/v-kids-watchneue-vodafone-smartwatch-verbindet-dich-mit-deinen-kindern/\#/ (letzter Zugriff am 26.11.2020).

Behnken, Imbke / Zinnecker, Jürgen (2019): Straße. In: Jürgen Hasse / Verena Schreiber (Hg.), Räume der Kindheit. Ein Glossar. Bielefeld: transcript, 329-335.

Belina, Bernd (2013): Raum. Münster: Westfälisches Dampfboot.

Blumer, Herbert (1981): Der methodologische Standort des symbolischen Interaktionismus. In: Arbeitsgruppe Bielefelder Soziologen (Hg.): Alltagswissen, Interaktion und gesellschaftliche Wirklichkeit Bd. 1. Opladen: Westdeutscher Verlag, 80-146.

Carver, Alison / Watson, Ben / Shaw, Ben / Hillman, Mayer (2013): A comparison study of children's independent mobility in England and Australia. In: Childrens Geographies $11 / 4,461-475$.

Degele, Nina (2002): Einführung in die Techniksoziologie. München: W. Fink.

Deutsches Institut für Vertrauen und Sicherheit im Internet (2015): DIVSI U9-Studie. Kinder in der digitalen Welt. Eine Grundlagenstudie des SINUS-Instituts Heidelberg 
im Auftrag des Deutschen Instituts für Vertrauen und Sicherheit im Internet (DIVSI). Hamburg. https://www.divsi.de/wp-content/uploads/2015/o6/U9-Studie-DIVSI-web. pdf (letzter Zugriff am 20.11.2020).

Donath, Andreas (2012): Schulkinder durch Funkchips erfassbar. https://www.golem.de/ news/rfid-schulkinder-durch-funkchips-erfassbar-1203-90726.html (letzter Zugriff am 20.11.2020).

Drüeke, Ricarda / Haug, Sonja / Keller, Wolfgang / Weber, Karsten (2007): Vertrauen ist gut, Kontrolle ist besser? - Privatsphäre und die Nutzung digitaler mobiler Endgeräte in interpersonalen Beziehungen. In: merz - medien + erziehung. Zeitschrift für Medienpädagogik 51/6, 25-35.

Egger, Rudolf / Hummel, Sandra (2016): Lernwelt Schulweg. Sozialräumliche Annäherungen an ein Alltagsphänomen. Wiesbaden: Springer VS.

Ema, Arisa / Fujigaki, Yuko (2011): How far can child surveillance go? Assessing the parental perceptions of an RFID child monitoring system in Japan. In: Surveillance \& Society 9/1-2, 132-148.

Fegter, Susann (2014): Mobilität - Technik - Geschlecht: Sozialisationsprozesse von Jungen und Mädchen in der Stadt. In: Berliner Debatte Initial 3, 20-33.

Fegter, Susann / Andresen, Sabine (2019): Erziehung und Bildung in der Kindheit. In: Fabian Kessl / Christian Reutlinger (Hg.), Handbuch Sozialraum. Wiesbaden: Springer VS, 401-418.

Feldhaus, Michael (2003): „Remote Control“ durch das Mobiltelefon - empirische Ergebnisse zu einer neuen Qualität in der Soziologie der Erziehung. In: Zeitschrift für Soziologie der Erziehung und Sozialisation 23/4, 416-432.

Fotel, Trine / Thomsen, Thyra Uth (2004): The surveillance of children's mobility. In: Surveillance \& Society 1/4, 535-554.

Frers, Lars / Krasmann, Susanne / Wehrheim, Jan (2013): Geopolicing und Kriminalitätskartierungen. Wie Polizeien sich ein Bild machen. In: Kriminologisches Journal 45/3, 166-179.

Furedi, Frank (2002): Paranoid parenting: Why ignoring the experts may be best for your child. Chicago: Chicago Review Press.

Ghosh, Arup Kumar / Badillo-Urquiola, Karla / Guha, Shion / LaViola Jr, Joseph J. / Wisniewski, Pamela J. (2018): Safety vs. surveillance: What children have to say about mobile apps for parental control. In: Regan Mandryk / Mark Hancock (Hg.), Engage with CHI. Proceedings of the 2018 CHI Conference on Human Factors in Computing Systems. New York: Association for Computing Machinery, 1-14.

Goold, Benjamin / Loader, Ian / Thumala, Angelica (2010): Consuming security? Tools for a sociology of security consumption. In: Theoretical Criminology 14/3: 3-30.

Greiner, Lena / Horchert, Judith (2018): Der Spion im Ranzen. In: Der Spiegel, 25.1.2018. https://www.spiegel.de/netzwelt/netzpolitik/schutzranzen-wolfsburg-stoppt-projektmit-peilsendern-an-schultaschen-a-1189624.html (letzter Zugriff am 25.11.2020).

Gryl, Inga (2019): Tracking. In: Jürgen Hasse / Verena Schreiber (Hg.), Räume der Kindheit. Ein Glossar. Bielefeld: transcript, 336-341.

Haggerty, Kevin D. / Ericson, Richard V. (2006): The new politics of surveillance and visibility. In: Kevin D. Haggerty / Richard V. Ericson (Hg.), The new politics of surveillance and visibility. Toronto $u$. a.: University of Toronto Press, 3-25.

Hoepken, Thorsten (2020a): Vodafone Curve: Brandneuer GPS-Tracker verbindet, was uns lieb und teuer ist. https://www.vodafone.de/newsroom/digitales-leben/vodafonecurve-brandneuer-gps-tracker-verbindet-was-uns-lieb-und-teuer-ist/ (letzter Zugriff am 26.11.2020).

Hoepken, Thorsten (2020b): Vodafone und Disney kooperieren bei der Entwicklung einer neuen Smart Watch für Kinder. https://www.vodafone.de/newsroom/digitales-leben/ vodafone-und-disney-kooperieren-bei-der-entwicklung-einer-neuen-smart-watch-fuerkinder/ (letzter Zugriff am 26.11.2020).

Jergus, Kerstin / Krüger, Jens Oliver / Roch, Anna (2018): Elternschaft zwischen Projekt und Projektion. Einleitung in den Band. In: Kerstin Jergus / Jens Oliver Krüger / Anna Roch (Hg.), Elternschaft zwischen Projekt und Projektion. Aktuelle Perspektiven der Elternforschung. Wiesbaden: Springer VS, 1-27.

Jurczyk, Karin / Schier, Michaela / Szymenderski, Peggy / Lange, Andreas / Voß, Günter (2009): Entgrenzte Arbeit - entgrenzte Familie. Berlin: edition sigma.

Kamin, Anna-Maria / Meister, Dorothee M. (2020): Familie und Medien. In: Jutta Ecarius / Anja Schierbaum (Hg.), Handbuch Familie Bd. 3. Wiesbaden: Springer Fachmedien, 1-19. 
Katz, Cindi (2001): The state goes home: Local hyper-vigilance of children and the global retreat from social reproduction. In: Social Justice 28/3, 47-56.

Kistler, Katharina (2015): Tracking-Apps. Wenn Eltern das Handy der Kinder ausspähen. In: Wirtschaftswoche, 9.7.2015. http://www.wiwo.de/technologie/digitale-welt/trackingapps-wenn-eltern-das-handy-der-kinder-ausspaehen/11998838-all.html (letzter Zugriff am 10.7.2020).

Krasmann, Susanne / Kreissl, Reinhard / Kühne, Sylvia / Paul, Bettina / Schlepper, Christina (2014): Die gesellschaftliche Konstruktion von Sicherheit. Zur medialen Vermittlung und Wahrnehmung der Terrorismusbekämpfung. Schriftenreihe Forschungsforum Öffentliche Sicherheit, Berlin.

Kühne, Sylvia (2019): Sanfte Überwachung? Eine Untersuchung zur Akzeptanz von digitalen Fingerabdrucktechnologien im Alltag. Dissertationsschrift Universität Hamburg. https:// ediss.sub.uni-hamburg.de/volltexte/2019/9995/pdf/Dissertation.pdf (letzter Zugriff am 26.11.2020).

Kutscher, Nadia (2008): Prävention unter Druck. Frühwarnsysteme und Elterntrainings. In: Sozial Extra 32/1-2, 38-41.

Lachance, Jocelyn (2020): Parental surveillance of teens in the digital era: the „ritual of confession” to the „ritual of repentance”. In: International Journal of Adolescence and Youth 25/1, 355-363.

LeMoyne, Terri / Buchanan, Tom (2011): Does „hovering” matter? Helicopter parenting and its effect on well-being. In: Sociological Spectrum 31/4, 399-418.

Lessenich, Stephan (2008): Die Neuerfindung des Sozialen. Der Sozialstaat im flexiblen Kapitalismus. Bielefeld: transcript.

Ludwigsburger Kreiszeitung (2018): Wirbel um neue Schulranzen-App. In: Ludwigsburger Kreiszeitung, 29.1.2018. https://www.lkz.de/lokales/stadt-ludwigsburg_artikel,-wirbelum-neue-schulranzen-app-_arid,462118.html (letzter Zugriff am 25.11.2020).

Lyon, David (2001): Surveillance society. Monitoring everyday life. Buckingham/Philadelphia: Open University Press.

Maas, Marie-Charlotte (2019): Familonet: Gründer kaufen Ortungs-App zurück. In: Wirtschaftswoche Gründer, 28.8.2019. https://gruender.wiwo.de/familonet-gruenderkaufen-ortungs-app-zurueck/ (letzter Zugriff am 25.11.2020).

Marx, Gary T. (2006): Soft surveillance: The growth of mandatory volunteerism in collecting personal information - „Hey buddy can you spare a DNA?”. In: Torin Monahan (Hg.), Surveillance and security. Technological politics and power in everyday life. New York/ London: Routledge, 37-56.

Marx, Gary T. / Steeves, Valerie (2010): From the beginning: Children as subjects and agents of surveillance. In: Surveillance \& Society 7/3, 192-230.

Mattel (2015): Hello Barbie Messaging Q\&A. http://hellobarbiefaq.mattel.com/wp-content/ uploads/2015/12/hellobarbie-faq-v3.pdf (letzter Zugriff am 26.11.2020).

de Montjoye, Yves-Alexandre / Radaelli, Laura / Singh, Vivek Kumar / Pentland, Alex S. (2015): Unique in the shopping mall. On the reidentifiability of credit card metadata. In: Science 347/6221, 536-539.

Nihlén Fahlquist, Jessica (2017): The ethical concerns of using GPS to track children. In: Emmeline Taylor / Tonya Rooney (Hg.), Surveillance futures. Social and ethical implications of new technologies for children and young people. London/New York: Routledge, 122-131.

Ohlheiser, Abby (2019): „Don’t leave campus”. Parents are now using tracking apps to watch their kids at college. In: The Washington Post, 22.10.2019. https://www.washingtonpost. com/technology/2019/10/22/dont-leave-campus-parents-are-now-using-tracking-appswatch-their-kids-college/ (letzter Zugriff am 26.11.2020).

Perez, Sarah (2020): Family tracking app Life36o launches „Bubbles”, a location-sharing feature inspired by teens on TikTok. https://techcrunch.com/2020/10/12/familytracking-app-life36o-launches-bubbles-a-location-sharing-feature-inspired-by-teenson-tiktok/ (letzter Zugriff am 26.11.2020).

Ponte, Cristina / Velicu, Anka / Simões, José Alberto / Lampert, Claudia (2018): Parental practices in the era of smartphones. In: Jane Vincent / Leslie Haddon (Hg.), Smartphone cultures. London: Routledge, 41-54.

Praschl, Peter (2015): Eine Barbie mit WLAN ist das Ende der Kindheit. In: Welt, 22.4.2015. www.welt.de/kultur/article139863883/Eine-Barbie-mit-WLAN-ist-das-Ende-derKindheit.html (letzter Zugriff am 25.11.2020).

Richter, Martina / Andresen, Sabine (2012): Orte „guter“ Kindheit? Aufwachsen im Spannungsfeld öffentlicher und privater Verantwortung. In: Andreas Lange / Regina 
Soremski (Hg.), Familie als Bildungswelt - Bildungswelt Familie. Schwerpunktheft der Zeitschrift für Soziologie der Erziehung und Sozialisation (ZSE) 32/3, 250-265.

Rocher, Luc / Hendrickx, Julien M. / de Montjoye, Yves-Alexandre (2019): Estimating the success of re-identifications in incomplete datasets using generative models. In: Nature Communication 10/1, 3069.

Röser, Jutta (2007): Medialisierter Beziehungsalltag. Wie im Medienhandeln Gemeinschaft und Rückzug gestaltet werden. In: Werner Faulstich (Hg.), Beziehungskulturen. München: Fink, 130-139.

Schetsche, Michael (1993): Sexualkontakte zwischen Erwachsenen und Kindern als soziales Problem. In: Soziale Probleme 4, 56-77.

Schreiber, Verena (2018): Kindheit. In: Bernd Belina / Matthias Naumann / Anke Strüver (Hg.), Handbuch Kritische Stadtgeographie. Münster: Westfälisches Dampfboot, 306-312.

Strüver, Anke (2018): Doreen Massey - Stadt und Geschlecht. In: Bernd Belina / Matthias Naumann / Anke Strüver (Hg.), Handbuch Kritische Stadtgeographie. Münster: Westfälisches Dampfboot, 42-47.

Taylor, Emmeline / Rooney, Tonya (2017): Digital playgrounds. Growing up in the surveillance age. In: Emmeline Taylor / Tonya Rooney (Hg.), Surveillance futures. Social and ethical implications of new technologies for children and young people. London/New York: Routledge, 1-16.

Williams, Stephen / Williams, Lynda (2005): Space invaders. The negotiation of teenage boundaries through the mobile phone. In: The Sociological Review 53/2, 314-331.

Wucherpfennig, Claudia (2019): Kinderzimmer. In: Jürgen Hasse / Verena Schreiber (Hg.), Räume der Kindheit. Ein Glossar. Bielefeld: transcript, 179-184.

Zeiher, Helga (1990): Organisation des Lebensraums bei Großstadtkindern - Einheitlichkeit oder Verinselung? In: Lothar Bertels / Ulf Herlyn (Hg.), Lebenslauf und Raumerfahrung. Opladen: Leske + Budrich, 35-58.

Zinnecker, Jürgen (1990): Vom Straßenkind zum verhäuslichten Kind. In: Imbke Behnken (Hg.), Stadtgesellschaft und Kindheit im Prozeß der Zivilisation. Wiesbaden: VS Verlag für Sozialwissenschaften, 142-162.

Zuboff, Shoshana (2018): Das Zeitalter des Überwachungskapitalismus. Frankfurt am Main/New York: Campus.

\section{Parental Control Technologies and the surveillance of children's mobility}

In the context of advancing (urban) technisation and digitalisation, a continuously growing market of tracking applications is establishing, enabling parents to find out the location of their children in real-time and track their movements. However, the mere availability of these parental control technologies does not explain their actual use. Nor does their definition as control and surveillance of children and adolescents result from the technologies themselves, but is being negotiated in interactions and practices. The article proposes hypotheses on why these technologies are increasingly used and what consequences this has for the individual and society and asks which implications this holds for urban research. 
